Research Article

\title{
Palmitic Acid Induces MicroRNA-221 Expression to Decrease Glucose Uptake in HepG2 Cells via the PI3K/AKT/GLUT4 Pathway
}

\author{
Fang Huang (D), Jie Chen, Jingwen Wang, Pingping Zhu, and Wenting Lin \\ School of Public Health, Fujian Medical University, Xueyuan Road 1, Minghou District, Fuzhou City, Fujian Province, China \\ Correspondence should be addressed to Fang Huang; huangfang@fjmu.edu.cn
}

Received 15 June 2019; Revised 23 August 2019; Accepted 4 October 2019; Published 11 November 2019

Academic Editor: Konstantinos Kantartzis

Copyright (c) 2019 Fang Huang et al. This is an open access article distributed under the Creative Commons Attribution License, which permits unrestricted use, distribution, and reproduction in any medium, provided the original work is properly cited.

\begin{abstract}
Obesity-related insulin resistance and high fatty acid concentrations occur during the development of type 2 diabetes mellitus. The role of high concentrations of plasma-free fatty acids is not fully understood. In this study, palmitic acid (PA, $0.8 \mathrm{mM}$ for $24 \mathrm{~h}$ ) induced the expression of miR-221 that bound to phosphoinositide 3-kinases (PI3K) mRNA to inhibit glucose uptake by HepG2 cells. Compared with controls, PA significantly decreased glucose uptake, increased insulin receptor substrate-2 (IRS-2) and miR221 expression, and decreased phosphoinositide 3-kinase (PI3K), protein kinase B (AKT), and glucose transporter type 4 (GLUT4) mRNA expression. Luciferase reporter assay revealed that miR-221 binding inhibited PI3K expression. Transfection of HepG2 cells with an miR-221 mimic induced miR-221 expression and inhibited the PI3K/AKT pathway. PA decreased glucose uptake in HepG2 cells by inducing the expression of miR-221, which bound to PI3K mRNA and suppressed PI3K/AKT signaling. miR-221 may be a novel target for preventing and treating obesity-induced insulin resistance.
\end{abstract}

\section{Introduction}

Obesity is a serious global health problem $[1,2]$. In the United States, the prevalence of youth and adult obesity is increasing, with an age-adjusted prevalence of $35 \%$ in men and $40.4 \%$ in women in 2013-2014 [3]. The prevalence of type 2 diabetes mellitus (T2DM) increases along with obesity and has an estimated prevalence of $18.5 \%$ in obese adults and $5.4 \%$ in normal-weight adults in the United States in 20132014 [4]. T2DM is a serious chronic metabolic disease triggered by impaired insulin signal pathways and systemic insulin resistance and the lack of response to insulin target cells such as hepatocytes, skeletal muscle cells, and adipocytes [5]. Insulin resistance occurs during the development of metabolic abnormalities and diseases, including T2DM, hypertension, and dyslipidemia, and decreasing insulin resistance improves metabolic control in T2DM patients [6].

Activated insulin receptor substrate-2 (IRS-2) regulates glucose homeostasis [7]. It transduces insulin action by stimulating the phosphoinositide 3-kinases/protein kinase B
(PI3K/AKT) pathway and promotes glucose uptake by insulin-sensitive glucose transporter type 4 (GLUT4) in the plasma membrane. Glucose transport fails because of insulin resistance in T2DM. Defective GLUT4 transport is a feature of insulin resistance, which is a precursor of T2DM [8]. MicroRNAs (miRNAs) are small noncoding RNA molecules that consist of approximately 23 nucleotide pairs [9]. They have been reported to influence adipogenesis and fat metabolism, and differential expression of miRNAs has been reported in tissues from obese versus nonobese people [10]. A significant correlation has been reported between obesity and increased risk of insulin resistance and T2DM [11]. The pathogenesis of insulin resistance is complex and not well understood, but free fatty acids (FFAs) may be involved [12]. An excess of lipids increases circulating FFAs and evokes insulin resistance in muscle and liver tissues [13]. Palmitic acid (PA), a representative FFA, has been shown to directly impair insulin signaling in cultured hepatocytes and myotubes [14]. Palmitic acid (PA) is the most common saturated fatty acid found in animals and plants and is found in foods 
like meat, cheese, butter, and other dairy products. PA or palmitate at concentrations of 0.4 to $1.0 \mathrm{mM}$ can induce a model of insulin resistance in cultured HepG2 cells [15-17]. Whether miRNAs are involved in the induction of resistance is not yet understood.

In this study, the molecular mechanism of PA-induced insulin resistance was investigated in HepG2 human hepatocyte cells. The aim was to develop novel rationale prevention and treatment of T2DM. We found that PA induced miR-221 expression in HepG2 cells that impaired PI3K/AKT signaling pathway and inhibited glucose uptake.

\section{Materials and Methods}

2.1. HepG2 Cell Culture and Glucose Uptake Experiment. HepG2 cells (ATCC, Manassas, VA, USA) were maintained in Dulbecco's modified Eagle medium (DMEM) supplemented with $10 \%$ FBS, 100 units $/ \mathrm{mL}$ streptomycin, and $100 \mu \mathrm{g} / \mathrm{mL}$ penicillin at $37^{\circ} \mathrm{C}$ in a humidified $5 \% \mathrm{CO}_{2}$ atmosphere. To assess the glucose uptake, HepG2 cells were transferred to 6-well plates and treated with $0-0.8 \mathrm{mM}$ PA for $24 \mathrm{~h}$. Glucose uptake was assayed by the glucose oxidase method (Bio-Rongsheng, Shanghai, China), following the kit manufacturer's instructions. Glucose oxidase catalyzes the breakdown of glucose to hydrogen peroxide and D-glucono$\delta$-lactone. Peroxidase then catalyzes the formation of a red quinone imide in a reaction involving hydrogen peroxide, 4aminoantipyrine, and phenol. The absorbance of the resulting solution at a wavelength of $490 \mathrm{~nm}$ is proportional to the glucose concentration.

\subsection{Quantitative Real-Time Polymerase Chain Reaction ( $q R T$ -} $P C R$ ). HepG2 cells were harvested after treatment and washed three times with ice-cold phosphate-buffered saline. Total RNA was extracted by TRIzol reagent (Invitrogen) following the manufacturer's instructions. The purity and concentration of the extracted RNA were determined at 260/ $280 \mathrm{~nm}$ using a spectrophotometer (NanoDrop ND-1000) and was reverse transcribed into cDNA with a TaKaRa OneStep RT-PCR kit using $1 \mu \mathrm{g}$ of total RNA and the supplied Oligo (dT) primers. Gene expression was determined by qRT-PCR performed with SYBR Green chemistry and a StepOnePlus system (LightCycler 480 II, Roche). The PCR program started at $95^{\circ} \mathrm{C}$ for $30 \mathrm{~s}$ followed by 40 cycles of denaturation at $95^{\circ} \mathrm{C}$ for $5 \mathrm{~s}$, annealing at $60^{\circ} \mathrm{C}$ for $30 \mathrm{~s}$, extension at $95^{\circ} \mathrm{C}$ for $5 \mathrm{~s}$, and a final extension at $60^{\circ} \mathrm{C}$ for $1 \mathrm{~min}$. Melting curves were obtained stepwise from $55^{\circ} \mathrm{C}$ to $95^{\circ} \mathrm{C}$. Data were reported as fold change $\left(2^{-\triangle \triangle \mathrm{Ct}}\right)$. Assays were performed independently in triplicate. The primer sequences are shown in Table 1 .

2.3. miRNA Isolation and Detection. A miRNA cDNA Synthesis Reaction System and a qPCR system $\left(\right.$ Mir- $^{\mathrm{TM}}$ miRNA First-Strand Synthesis and SYBR ${ }^{\circledR}$ qRT-PCR, TaKaRa Bio Company, USA) were used, following the manufacturer's instructions. The prepared PCR solution, except for the DNA template, was pipetted into 96-well plates. The DNA template of the PCR was added, and the amplification conditions were set. The amplification reaction was performed with a QuantStudio ${ }^{\mathrm{TM}} 3$ and 5 Real-Time PCR System with U6 as a control. The reactions were performed in triplicate. The primer sequences for miRNA-221 and U6 are shown in Table 2.

2.4. Luciferase Reporter Assay. Psi-Check2 wild-type $3^{\prime}$ untranslated sequences (wt-3'-UTR) of PI3KR1, containing the miR-221 binding site ligated to a pLuc-reporter luciferase vector, are shown in Supplementary Figure 1 and were synthesized by Sangon Biotech (Shanghai, China). PCR was performed with Primer STAR ${ }^{\circledR}$ HS DNA Polymerase (TaKaRa Bio Inc. Shiga, Japan) following the manufacturer's instructions. Psi-Check2 mutated vectors (mut-3'UTR) were constructed using the Fast Mutagenesis System (TransGen Biotech, China). All constructs were verified by DNA sequencing.

HEK-293T human embryonic kidney cells were seeded in 24-well plates, cotransfected with $10 \mathrm{nmol}$ pre-miR-221 or pre-miR-NC and $100 \mathrm{ng}$ pLuc-3'-UTR. The cells were harvested $24 \mathrm{~h}$ after transfection. Luciferase activity was measured with a Dual-Luciferase Reporter Assay System (Promega, USA) and a Glomax Luminometer (Promega, USA). Renilla luciferase activity was normalized to firefly luciferase. All assays were conducted in triplicate.

2.5. Cell Transfection. Cells were transfected with Lipofectamine 3000 (Thermo Fisher Scientific, Waltham, MA, USA), following the manufacturer's instructions. The miR221 mimic and mi-221 inhibitor were purchased from Sangon Biotech (Shanghai, China). The primer sequences are shown in Table 3. PA was added to the cell culture media $24 \mathrm{~h}$ after transfection, and cells were cultured for another $24 \mathrm{~h}$.

2.6. Western Blotting. Total protein was extracted with RIPA lysis buffer and separated by sodium dodecyl sulfate-polyacrylamide gel electrophoresis (SDS-PAGE). The proteins were transferred to polyvinylidene difluoride membranes, blocked with $5 \%$ skim milk in Tris-buffered saline containing $0.1 \%$ Tween-20 for $1 \mathrm{~h}$, and then incubated with anti-PI3K, anti-AKT, anti-p-PI3K, anti-p-AKT, and anti- $\beta$-actin primary antibodies (Proteintech, Rosemont, IL, USA) for $16 \mathrm{~h}$ at $4^{\circ} \mathrm{C}$. Membranes were washed three times in Tris-buffered saline containing $0.1 \%$ Tween-20 and then incubated with anti-mouse or anti-rabbit IgG secondary antibodies for $1 \mathrm{~h}$. Immunoreactive bands were visualized by a commercial electrochemiluminescence (ECL) kit (Amersham Pharmacia Biotech, Little Chalfont, Great Britain).

2.7. Statistical Analysis. Values were expressed as the means \pm standard deviation (SD). Between-group differences were compared with unpaired two-tailed $t$-tests. Multigroup differences were compared by analysis of variance with Dunnett's multiple comparison test or the Tukey-Kramer test. $p$ values $<0.05$ were considered statistically significant. 
TABLE 1: The primer sequences of IRS-2, PI3K, and GLUT4.

\begin{tabular}{lcc}
\hline Gene & & Primer sequence \\
& Forward & Reverse \\
\hline IRS-2 & CACCTACGCCAGCATTGACTTC & CAAACACAGTCATTGCTCAGATCC \\
PI3K & AGCATTGGGACCTCACATTACACA & ACTGGAAACACAGTCCATGCACATA \\
AKT & AGCGACGTGGCTATTGTGAA & CACGTTGGTCCACATCCTG \\
GLUT4 & GGGCTGAGACAGGGACCATAAC & CATGAGCAATGGCATCCCAGAA \\
$\beta$-Actin & TGGATCAGCAAGCAGGAGTA & ATGGTGGTGAAGACGCCAGTA \\
\hline
\end{tabular}

TABle 2: The primer sequences of U6 and miR-221.

\begin{tabular}{lcc}
\hline $\begin{array}{l}\text { Name of } \\
\text { miRNA }\end{array}$ & \multicolumn{1}{c}{ Sequence of primer } \\
\hline Hsa-miR-221- & Forward & CTACATTGTCTGCTGGGTTTC \\
$3 \mathrm{p}$ & $\begin{array}{l}\text { Forward } \\
\text { Reverse }\end{array}$ & TGGACGATACAGAGAAGATTAGC \\
& TGATTCACGAATTTGCG \\
\hline
\end{tabular}

TABLE 3: The sequences of miR-221-3p-mimic/inhibitor.

\begin{tabular}{lc}
\hline Name of miRNA & \multicolumn{1}{c}{ Sequence of primer } \\
\hline $\begin{array}{l}\text { Hsa-miR-221-3p- } \\
\text { mimic }\end{array}$ & AGCUACAUUGUCUGCUGGGUUUC \\
$\begin{array}{l}\text { Has-miR-221-3p- } \\
\text { inhibitor }\end{array}$ & GAAACCCAGCAGACAAUGUAGCU \\
\hline
\end{tabular}

\section{Results}

3.1. PA Decreased Glucose Uptake in HepG2 Cells. PA has been shown to directly impair insulin signaling in cultured hepatocytes and has been previously used to induce insulin resistance in HepG2 cells $[15,16]$. As shown in Figure 1, treatment with 0.2 to $0.8 \mathrm{mM}$ PA for $24 \mathrm{~h}$ did not inhibit cell viability and the lowest glucose uptake occurred with $0.8 \mathrm{mM}$ PA. Consequently, $0.8 \mathrm{mM}$ PA for $24 \mathrm{~h}$ was used to establish the HepG2 insulin resistance model.

3.2. PA Impairs PI3K/AKT Signaling Pathway and Increases miR-221 Expression. The PI3K/AKT pathway regulates glucose homeostasis via insulin-sensitive GLUT4 [18]. In this study, inhibition of the PI3K/AKT pathway was determined by assay of IRS-2, PI3K, AKT, and GLUT4 mRNA expression in HepG2 cells, before and after exposure to PA for $24 \mathrm{~h}$. IRS-2 mRNA expression was significantly increased by PA, but PI3K, AKT, and GLUT4 mRNA expressions were decreased. Normally, PI3K is upregulated by IRS-2, but that was not observed in this study, possibly because of the change in miRNA expression [19].

miRNAs regulate gene expression posttranscriptionally by directly binding to the $3^{\prime}$ - untranslated region $\left(3^{\prime}\right.$-UTR) of target mRNAs [9]. Dysregulation of miRNAs has previously been implicated in the pathogenesis of various diseases, including obesity and diabetes [20]. miR-221 is involved in the development of obesity and has been reported to negatively regulate insulin sensitivity [21]. qPCR confirmed that PA significantly increased miR-221 expression (Figure 2(b)).
To verify that PI3K was a direct target of miR-221, the putative miR-221-binding sequence at the $3^{\prime}$-UTR of the PI3K gene was subcloned into a luciferase reporter vector. Ectopic expression of miR-221 significantly reduced luciferase activity in HepG2 cells transfected with the reporter vector containing the PI3K $3^{\prime}$-UTR sequence. The effect of miR-221 was abolished when the binding sequence was mutated (Figure 2(c)).

3.3. miR-221 Expression Decreases Glucose Uptake in HepG2 Cells. The involvement of miR-221 in glucose uptake by HepG 2 cells treated with PA was investigated in HepG2 cells transfected with miR-221 mimic and inhibitor. The expression of miR-221 was significantly increased in HepG2 cells transfected by mimic and was decreased in those transfected by inhibitor with (Figure 3(a)) and without (Figure 3(b)) PA treatment. As shown in Figure 3(c), miR221 mimic significantly reduced glucose transport by HepG2 cells and miR-221 inhibitor reversed the reduction in response to PA, indicating that miR-221 modulated glucose uptake.

3.4. miR-221 Suppresses GLUT4 Expression via the PI3K/AKT Pathway. Changes in protein expression indicated that the effects of miR-221 on glucose transport were mediated by the PI3K/AKT pathway. Figure 4 shows that PI3K, p-PI3K, AKT, p-AKT, and GLUT4 protein expressions were decreased by miR-221 mimic and that the miR-221 inhibitor blocked the effects of PA on protein expression. The results indicate that miR-221 regulated glucose uptake via the PI3K/ AKT pathway.

\section{Discussion}

Overweight and obesity are both increasing in prevalence worldwide. Most patients with T2DM are overweight or obese and have elevated plasma FFA levels that are correlated with the severity of insulin resistance [22, 23]. Consumption of a diet high in saturated versus monounsaturated fats has been associated with increased insulin resistance [24]. High levels of circulating FFAs are thought to have a key role in initiating and promoting the progression of insulin resistance, but the mechanism of action is not fully understood. In this study, PA, a representative FFA, induced the expression of miR-221, which then bound to PI3K mRNA to inhibit its expression, and that of PI3K/ AKT pathway-associated proteins to decrease glucose uptake. 


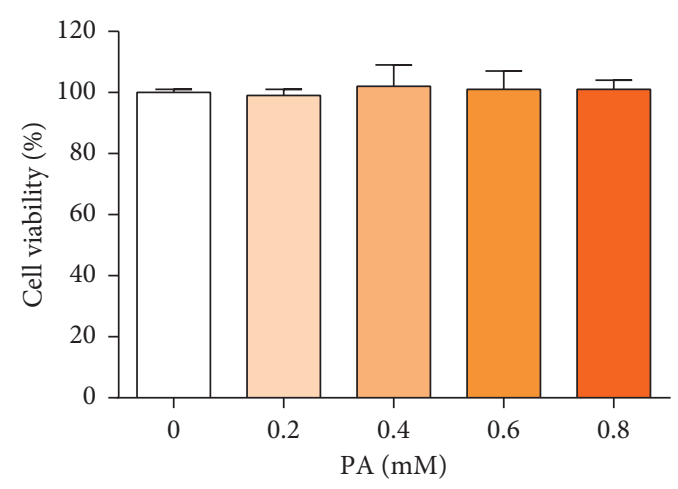

(a)

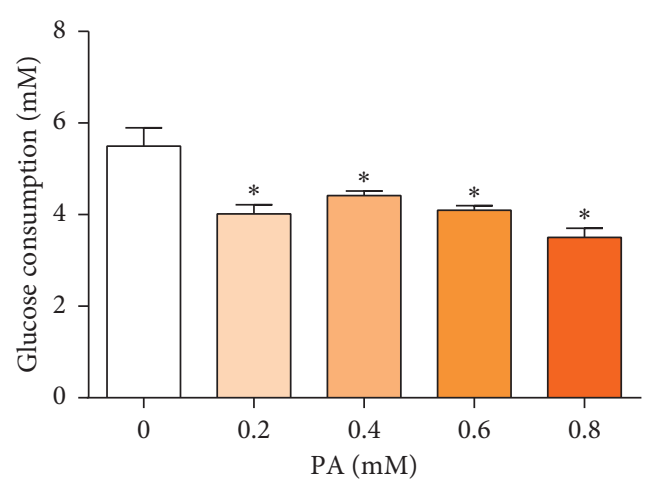

(b)

Figure 1: Effects on HepG2 cell viability (a) and glucose uptake (b) after treatment with $0-0.8 \mathrm{mM}$ PA treatment for $24 \mathrm{~h}$. Values are the means $\pm \mathrm{SD}(n=3) .{ }^{*} p<0.05$, versus $0 \mathrm{mM}$ PA.

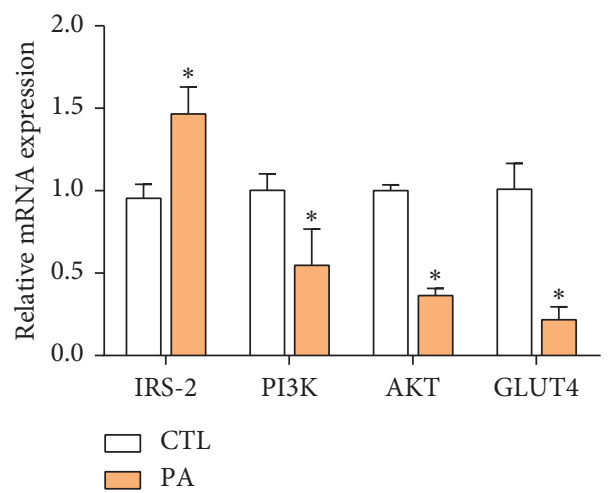

(a)

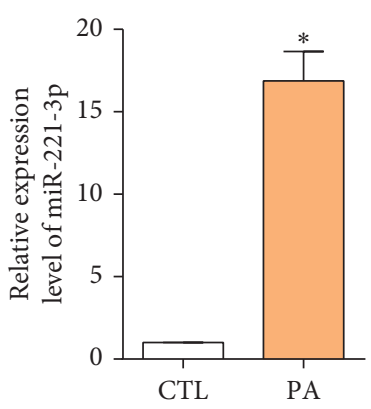

(b)

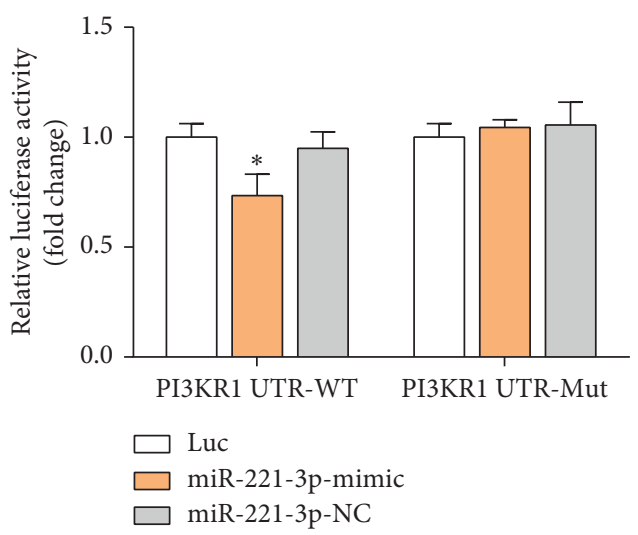

(c)

FIGURE 2: Effect of PA on the expression of PI3K/AKT-related mRNAs and miR-221. IRS-2, PI3K, AKT, and GLUT4 mRNA expressions were assayed in HepG2 cells after treatment with $0.8 \mathrm{mM}$ PA for $24 \mathrm{~h}$ (a); PA significantly increased miR-221 expression compared with controls (b); luciferase reporter assay demonstrated PI3K binding of miR-221 by PI3K (c). Values are the means $\pm \mathrm{SD}(n=3)$. ${ }^{*} p<0.05$, versus control ( $a$ and $b$ ) or UTR-Mut (c).

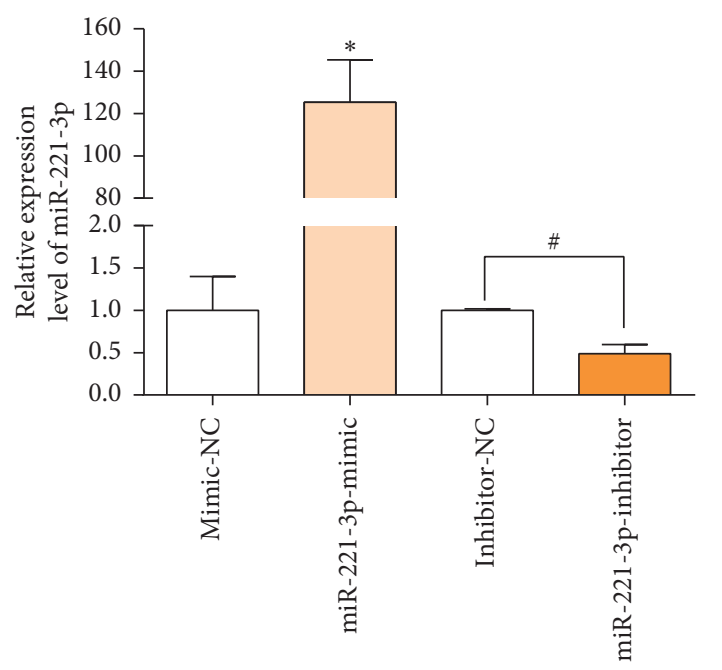

(a)

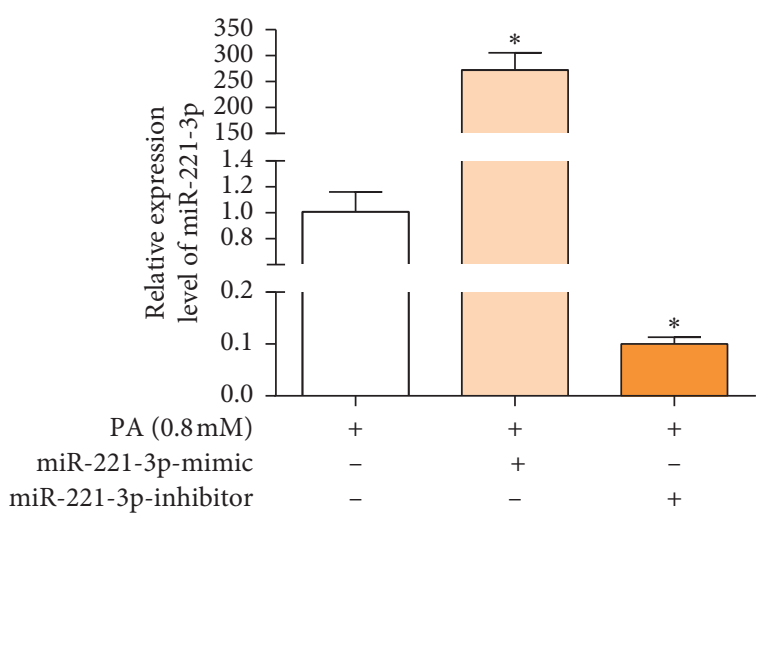

(b)

Figure 3: Continued. 


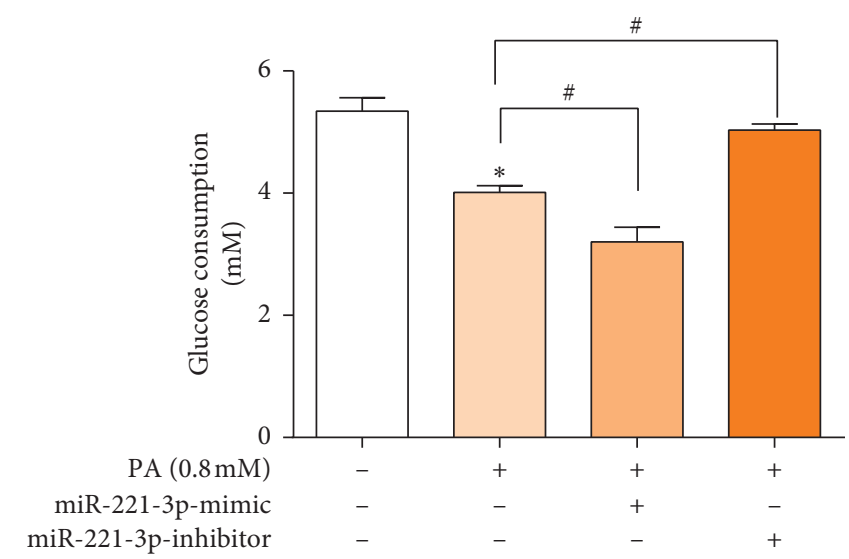

(c)

FIGURE 3: Effect of miR-221 mimic and inhibitor on miR-221 transfection on miRNA expression and glucose uptake. miR-221 expression was assayed by qPCR. (a); HepG cells were transfected with miR-221 mimic or inhibitor for $24 \mathrm{~h} \mathrm{PA}$ was added to culture media for $24 \mathrm{~h}$ and miR-221 expression was assayed (b); glucose consumption was determined by the glucose oxidase method (c). Values are the means \pm SD $(n=3) .{ }^{*} p<0.05$, versus control group, ${ }^{\#} p<0.05$, versus the indicated group $(n=3)$.

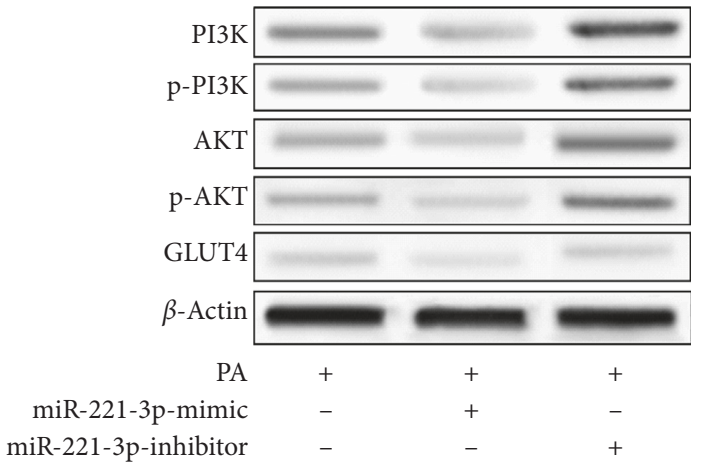

(a)

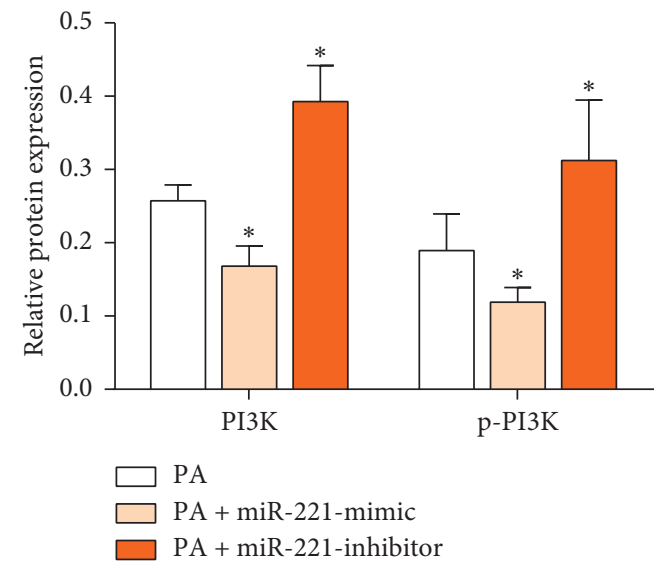

(b)

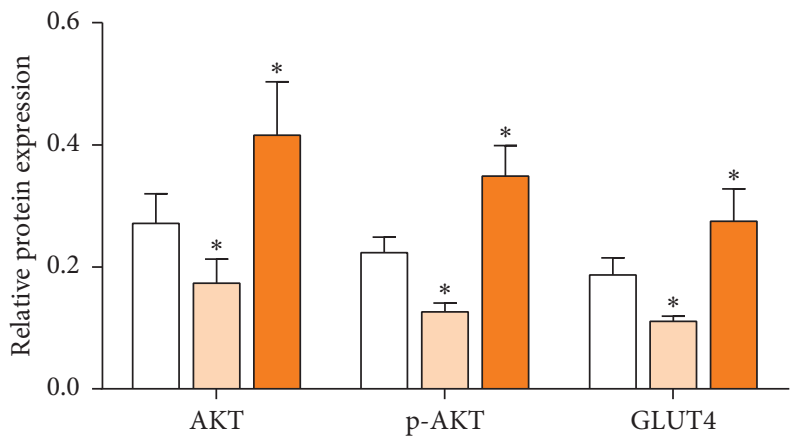

(c)

Figure 4: Effect of miR-221 mimic and inhibitor on expression of PI3K/AKT pathway proteins. HepG cells were transfected with miR-221 mimic or inhibitor for $24 \mathrm{~h}$; PA was then added for an additional $24 \mathrm{~h}$. PI3K, p-PI3K, AKT, p-AKT, and GLUT4 protein expressions were assayed by immunoblotting (a), normalized against $\beta$-actin, and expressed as fold change compared with PA-treated controls (b, c). Values are the means $\pm \mathrm{SD}(n=3) .{ }^{*} p<0.05$, versus control group.

IRS-2 is a cytoplasmic adaptor protein that organizes signaling complexes downstream of cell surface receptors and it coordinates responses to insulin that are associated with induction and worsening of resistance [25]. PI3K is an intracellular signal transducer of diverse cellular functions. $\mathrm{AKT}$ is a protein kinase downstream of PI3K that mediates the release of growth factors, cytokines, and other stimuli of cell survival, growth, proliferation, angiogenesis, and 


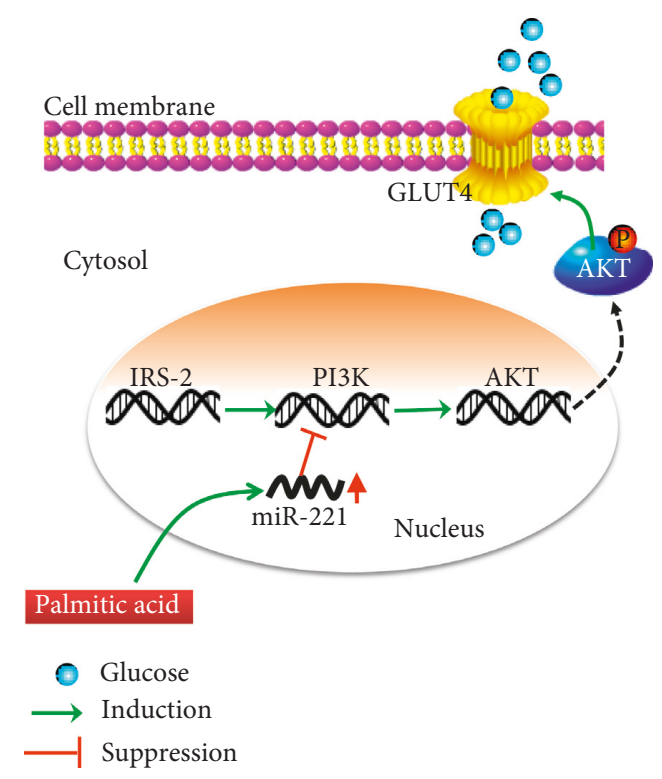

FIGURE 5: Proposed mechanism of PA induction of insulin resistance in HepG2 cells. PA induces miR-221 overexpression. miR221 binds PI3K to decrease PI3K and AKT expression and phosphorylation. Impairment of the PI3K/AKT pathway results in reduced GLUT4 expression and glucose consumption, which induces insulin resistance.

metabolism. The IRS/PI3K/AKT signaling pathway regulates insulin signaling and lipid metabolism $[19,26]$. In this study, PA induced the expression of IRS-2 but not PI3K, its downstream target. The lack of PI3K response was mediated by miR-221.

There is evidence that miRNAs are involved in insulin secretion, $\beta$-cell differentiation, and glycolipid metabolism and that they contribute to the progression of T2DM $[27,28]$. Previous studies have described the involvement of miR-221 in the development of obesity and decreased insulin sensitivity $[21,29]$. In the previous study, postnatal overfeeding was found to induce miR-221 expression and impaired PI3K/AKT signaling in the livers of adult male rats. In the present study, we found that miR-221 influenced IRS/ $\mathrm{PI} 3 \mathrm{~K} / \mathrm{AKT}$ signaling during the induction of insulin resistance by PA.

miR-221 is located on the $\mathrm{X}$ chromosome (Xp11.3). It is highly conserved in vertebrates and is a putative oncogene that is overexpressed in a number of human tumors [30-32]. Adipose miR-221 is upregulated in obesity, and its expression is positively correlated with an increased body mass index in the Pima Indian population, which has a high prevalence of T2DM [33]. The luciferase reporter assay revealed that in HepG2 cells transfected by miR-221 mimic and inhibitor, PA induced the expression of miR-221 by binding to PI3K mRNA and suppressing its expression. Thus, miR-221 played an important role in the insulin resistance induced by PA.

GLUT4 is an insulin-regulated transmembrane glucose transporter that controls glucose homeostasis and is a downstream target of the PI3K/AKT pathway [18]. In this study, PA decreased glucose uptake by suppressing GLUT4 expression. The decreased GLUT4 expression can be accounted for by the impairment of the PI3K/AKT signaling pathway. Polysaccharides extracted from Enteromorpha prolifera, an edible seaweed, have hypoglycemic and hypolipidemic effects and have been shown to lower blood glucose by increasing GLUT4 expression [34-38]. Thus, polysaccharides from dietary seaweed may be found effective for preventing T2DM.

In summary, PA decreased glucose uptake in HepG2 cells by promoting miR-221 overexpression, which reduced GLUT4 expression and glucose uptake by impairing PI3K/ AKT signaling. A proposed mechanism of miR-221 overexpression induced by PA and the resulting impairment of the PI3K/AKT pathway is shown in Figure 5. The study results suggest that inhibition of miR-221 may have potential as a novel approach for preventing and treating obesityinduced insulin resistance.

\section{Abbreviations}

IRS-2: Insulin receptor substrate-2

PI3K: $\quad$ Phosphoinositide 3-kinase

AKT: $\quad$ Protein kinase B

GLUT4: $\quad$ Glucose transporter type 4

MicroRNA: miRNA

T2DM: $\quad$ Type 2 diabetes mellitus.

\section{Data Availability}

The data used to support the findings of this study are available from the corresponding author upon request.

\section{Conflicts of Interest}

The authors declare no conflicts of interest.

\section{Authors' Contributions}

Fang Huang and Jie Chen contributed equally to this work.

\section{Acknowledgments}

This study was supported by the Natural Science Foundation of Fujian Province (no. 2017J01810) and Special Funds for the Development of Local Colleges and Universities Supported by the Central Government (nos. 1003-03900134 and GW-201601).

\section{Supplementary Materials}

Supplementary Figure 1: a schematic diagram showing the construction of the luciferase reporter vectors. (Supplementary Materials)

\section{References}

[1] Y. Haidar and B. Cosman, "Obesity epidemiology," Clinics in Colon and Rectal Surgery, vol. 24, no. 4, pp. 205-210, 2011.

[2] L. Jing, C. M. Binkley, J. D. Suever et al., "Cardiac remodeling and dysfunction in childhood obesity: a cardiovascular 
magnetic resonance study," Journal of Cardiovascular Magnetic Resonance, vol. 18, no. 1, p. 28, 2016.

[3] E. J. Benjamin, M. J. Blaha, S. E. Chiuve et al., "Heart disease and stroke statistics-2017 update: a report from the American heart association," Circulation, vol. 135, no. 10, pp. e146-e603, 2017.

[4] S. Saydah, K. M. Bullard, Y. Cheng et al., "Trends in cardiovascular disease risk factors by obesity level in adults in the United States, NHANES 1999-2010," Obesity, vol. 22, no. 8, pp. 1888-1895, 2014.

[5] A. R. Saltiel, "New perspectives into the molecular pathogenesis and treatment of type 2 diabetes," Cell, vol. 104, no. 4 , pp. 517-529, 2001.

[6] A. A. Tahrani, C. J. Bailey, S. Del Prato, and A. H. Barnett, "Management of type 2 diabetes: new and future developments in treatment," The Lancet, vol. 378, no. 9786, pp. 182-197, 2011.

[7] J. L. Evans, B. A. Maddux, and I. D. Goldfine, "The molecular basis for oxidative stress-induced insulin resistance," Antioxidants \& Redox Signaling, vol. 7, no. 7-8, pp. 1040-1052, 2005.

[8] R. A. DeFronzo and D. Tripathy, "Skeletal muscle insulin resistance is the primary defect in type 2 diabetes," Diabetes Care, vol. 32, no. 2, pp. S157-S163, 2009.

[9] D. P. Bartel, "MicroRNAs: target recognition and regulatory functions," Cell, vol. 136, no. 2, pp. 215-233, 2009.

[10] T. Fu, S.-E. Choi, D.-H. Kim et al., "Aberrantly elevated microRNA-34a in obesity attenuates hepatic responses to FGF19 by targeting a membrane coreceptor -Klotho," Proceedings of the National Academy of Sciences, vol. 109, no. 40, pp. 16137-16142, 2012.

[11] S. E. Kahn, R. L. Hull, and K. M. Utzschneider, "Mechanisms linking obesity to insulin resistance and type 2 diabetes," Nature, vol. 444, no. 7121, pp. 840-846, 2006.

[12] V. T. Samuel, K. F. Petersen, and G. I. Shulman, "Lipid-induced insulin resistance: unravelling the mechanism," The Lancet, vol. 375, no. 9733, pp. 2267-2277, 2010.

[13] R. J. Perry, V. T. Samuel, K. F. Petersen, and G. I. Shulman, "The role of hepatic lipids in hepatic insulin resistance and type 2 diabetes," Nature, vol. 510, no. 7503, pp. 84-91, 2014.

[14] D. Gao, S. Nong, X. Huang et al., "The effects of palmitate on hepatic insulin resistance are mediated by NADPH oxidase 3derived reactive oxygen species through JNK and p38MAPK pathways," Journal of Biological Chemistry, vol. 285, no. 39, pp. 29965-29973, 2010.

[15] L. Chen, H. Teng, and H. Cao, "Chlorogenic acid and caffeic acid from Sonchus oleraceus Linn synergistically attenuate insulin resistance and modulate glucose uptake in HepG2 cells," Food and Chemical Toxicology, vol. 127, pp. 182-187, 2019.

[16] M. Ishii, A. Maeda, S. Tani, and M. Akagawa, "Palmitate induces insulin resistance in human HepG2 hepatocytes by enhancing ubiquitination and proteasomal degradation of key insulin signaling molecules," Archives of Biochemistry and Biophysics, vol. 566, pp. 26-35, 2015.

[17] T. Charytoniuk, N. Iłowska, K. Berk, K. Drygalski, A. Chabowski, and K. Konstantynowicz-Nowicka, "The effect of enterolactone on sphingolipid pathway and hepatic insulin resistance development in HepG2 cells," Life Sciences, vol. 217, pp. 1-7, 2019.

[18] H. Sano, L. Eguez, M. N. Teruel et al., "Rab10, a target of the AS160 Rab GAP, is required for insulin-stimulated translocation of GLUT4 to the adipocyte plasma membrane," Cell Metabolism, vol. 5, no. 4, pp. 293-303, 2007.
[19] B. D. Manning and L. C. Cantley, "AKT/PKB signaling: navigating downstream,” Cell, vol. 129, no. 7, pp. 1261-1274, 2007.

[20] V. Rottiers and A. M. Näär, "MicroRNAs in metabolism and metabolic disorders," Nature Reviews Molecular Cell Biology, vol. 13, no. 4, pp. 239-250, 2012.

[21] J. Peng, Y. Zhou, Z. Deng et al., "miR-221 negatively regulates inflammation and insulin sensitivity in white adipose tissue by repression of sirtuin-1 (SIRT1)," Journal of Cellular Biochemistry, vol. 119, no. 8, pp. 6418-6428, 2018.

[22] H. Liang, P. Tantiwong, A. Sriwijitkamol et al., "Effect of a sustained reduction in plasma free fatty acid concentration on insulin signalling and inflammation in skeletal muscle from human subjects," The Journal of Physiology, vol. 591, no. 11, pp. 2897-2909, 2013.

[23] J. P. H. Wilding, "The importance of free fatty acids in the development of Type 2 diabetes," Diabetic Medicine, vol. 24, no. 9, pp. 934-945, 2007.

[24] J. E. Galgani, R. D. Uauy, C. A. Aguirre, and E. O. Díaz, "Effect of the dietary fat quality on insulin sensitivity," British Journal of Nutrition, vol. 100, no. 3, pp. 471-479, 2008.

[25] R. A. Haeusler and D. Accili, "The double life of Irs," Cell Metabolism, vol. 8, no. 1, pp. 7-9, 2008.

[26] M. Mendez-Pertuz, P. Martinez, C. Blanco-Aparicio et al., "Modulation of telomere protection by the PI3K/AKT pathway," Nature Communications, vol. 8, no. 1, p. 1278, 2017.

[27] X. Li, "MiR-375, a microRNA related to diabetes," Gene, vol. 533, no. 1, pp. 1-4, 2014.

[28] C. Guay, E. Roggli, V. Nesca, C. Jacovetti, and R. Regazzi, "Diabetes mellitus, a microRNA-related disease?," Translational Research, vol. 157, no. 4, pp. 253-264, 2011.

[29] H. Xie, B. Lim, and H. F. Lodish, "MicroRNAs induced during adipogenesis that accelerate fat cell development are downregulated in obesity," Diabetes, vol. 58, no. 5, pp. 1050-1057, 2009.

[30] S. Galardi, N. Mercatelli, E. Giorda et al., "miR-221 and miR222 expression affects the proliferation potential of human prostate carcinoma cell lines by targeting p27Kip1," Journal of Biological Chemistry, vol. 282, no. 32, pp. 23716-23724, 2007.

[31] J.-J. Zhao, J. Lin, H. Yang et al., "MicroRNA-221/222 negatively regulates estrogen receptora and is associated with tamoxifen resistance in breast cancer," Journal of Biological Chemistry, vol. 283, no. 45, pp. 31079-31086, 2008.

[32] M. Frenquelli, M. Muzio, C. Scielzo et al., "MicroRNA and proliferation control in chronic lymphocytic leukemia: functional relationship between miR-221/222 cluster and p27," Blood, vol. 115, no. 19, pp. 3949-3959, 2010.

[33] A. Meerson, M. Traurig, V. Ossowski, J. M. Fleming, M. Mullins, and L. J. Baier, "Human adipose microRNA-221 is upregulated in obesity and affects fat metabolism downstream of leptin and TNF- $\alpha$," Diabetologia, vol. 56, no. 9, pp. 1971-1979, 2013.

[34] R. Ren, J. Gong, Y. Zhao et al., "Sulfated polysaccharide from Enteromorpha prolifera suppresses SREBP-1c and ACC expression to lower serum triglycerides in high-fat diet-induced hyperlipidaemic rats," Journal of Functional Foods, vol. 40, pp. 722-728, 2018.

[35] R. Ren, J. Gong, Y. Zhao, X. Zhuang, Y. Ye, and W. Lin, "Sulfated polysaccharides from Enteromorpha prolifera suppress SREBP-2 and HMG-CoA reductase expression and attenuate non-alcoholic fatty liver disease induced by a highfat diet," Food \& Function, vol. 8, no. 5, pp. 1899-1904, 2017.

[36] R. Ren, Z. Yang, A. Zhao et al., "Sulfated polysaccharide from Enteromorpha prolifera increases hydrogen sulfide 
production and attenuates non-alcoholic fatty liver disease in high-fat diet rats," Food \& Function, vol. 9, no. 8, pp. 4376-4383, 2018.

[37] Q. Wu, Q. Wang, J. Fu, and R. Ren, "Polysaccharides derived from natural sources regulate triglyceride and cholesterol metabolism: a review of the mechanisms," Food \& Function, vol. 10, no. 5, pp. 2330-2339, 2019.

[38] W. Lin, W. Wang, D. Liao et al., "Polysaccharides from Enteromorpha prolifera improve glucose metabolism in diabetic rats," Journal of Diabetes Research, vol. 2015, Article ID 675201, 12 pages, 2015. 


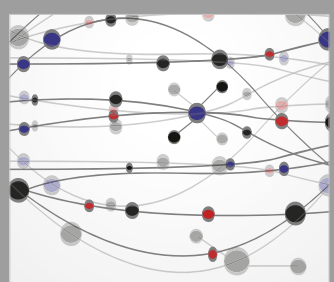

The Scientific World Journal
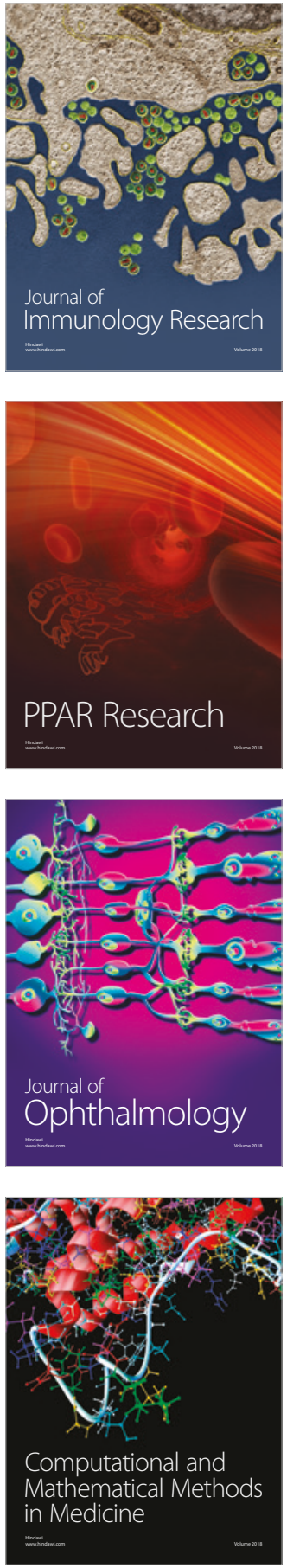

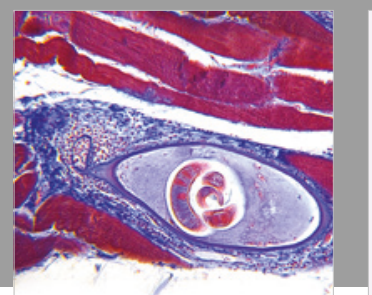

Gastroenterology Research and Practice

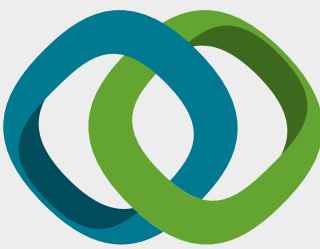

\section{Hindawi}

Submit your manuscripts at

www.hindawi.com
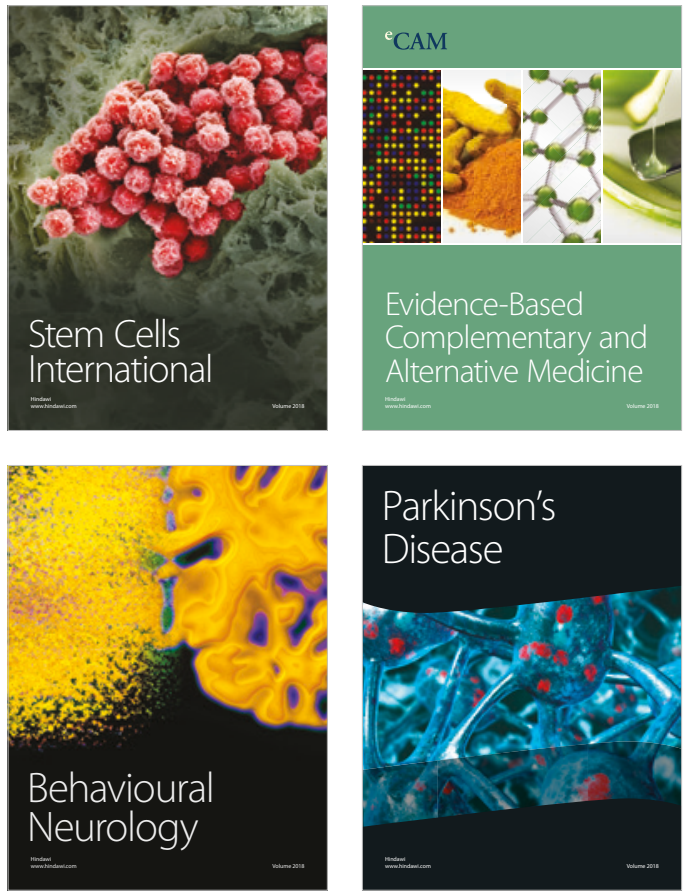

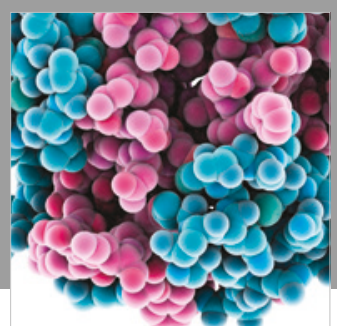

ournal of

Diabetes Research

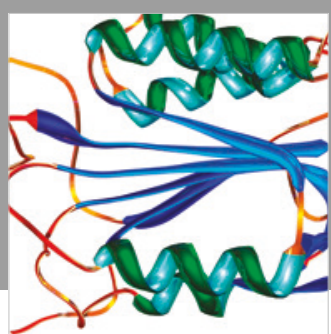

Disease Markers
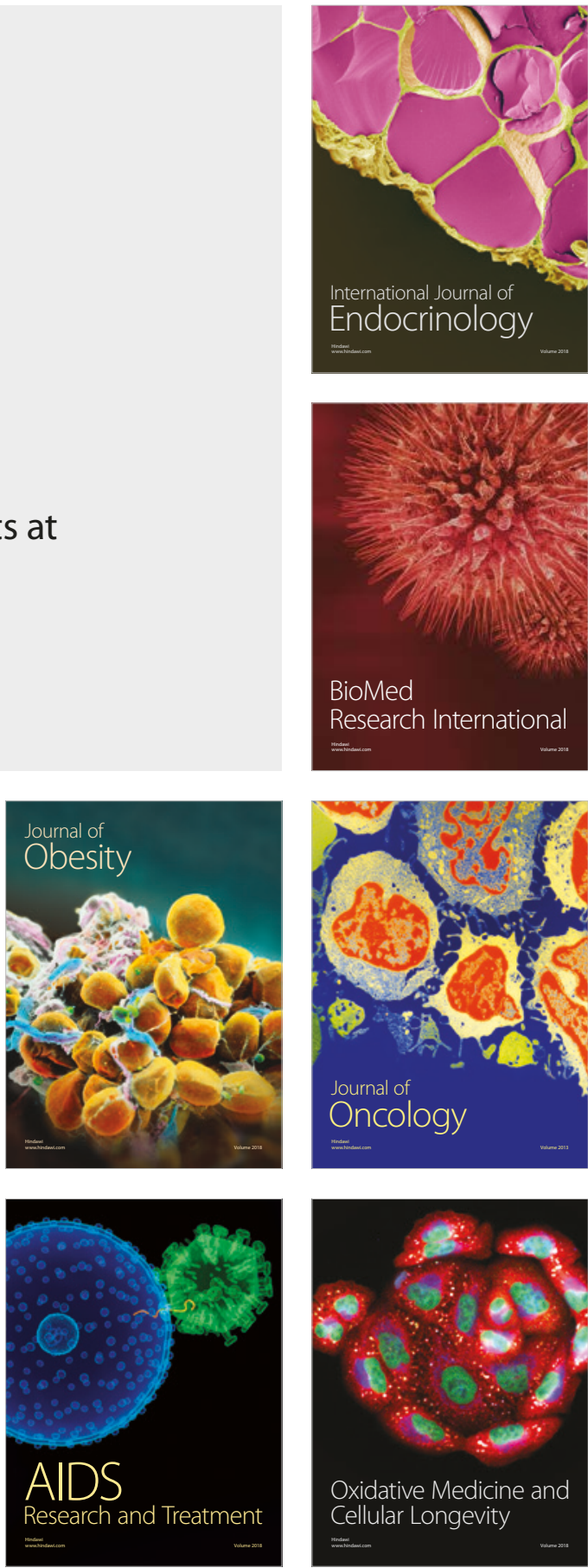